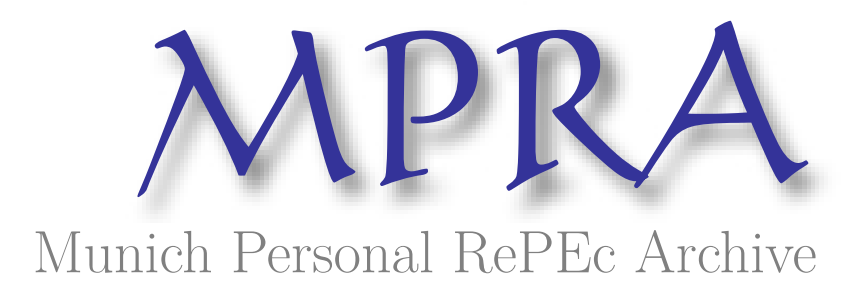

\title{
Culture and Stages of Economic Development
}

\author{
Chu, Angus C. and Kou, Zonglai and Wang, Xilin \\ University of Liverpool, Fudan University, Fudan University
}

March 2021

Online at https://mpra.ub.uni-muenchen.de/106900/

MPRA Paper No. 106900, posted 31 Mar 2021 11:35 UTC 


\title{
Culture and Stages of Economic Development
}

\author{
Angus C. Chu Zonglai Kou Xilin Wang
}

March 2021

\begin{abstract}
How do cultural differences in preferences affect economic development? This study develops a simple growth model that features two stages of development. In the first stage, economic growth is driven by human capital accumulation. In the second stage, economic growth is driven by innovation. The economy does not necessarily experience the transition from the first stage to the second stage. If this endogenous transition does not occur, the economy converges to a steady-state level of output. The economy remains in this middle-income trap if leisure preference is too strong or parental preference for education is either too weak or too strong.
\end{abstract}

JEL classification: O30, O40

Keywords: culture, education, innovation, economic development, middle-income trap

Chu: angusccc@gmail.com. Management School, University of Liverpool, Liverpool, United Kingdom. Kou: zlkou@fudan.edu.cn. China Center for Economic Studies, School of Economics, Fudan University, Shanghai, China. Wang: xilinwang@fudan.edu.cn. China Center for Economic Studies, School of Economics, Fudan University, Shanghai, China. 


\section{Introduction}

Culture is a set of values that influence people's preferences. To explore how cultural differences in preferences affect economic development, we develop a simple growth model that features two stages of development. In the first stage, economic growth is driven by human capital accumulation. In the second stage, economic growth is driven by innovation. The transition from the first stage to the second stage is endogenous. If this transition occurs, the economy converges to a balanced growth path with long-run growth driven by innovation. If the transition does not occur, the economy converges to a steady state with a stationary level of output. The economy remains in this middle-income trap if (1) leisure preference is too strong, (2) research productivity or education productivity is too low, or (3) parental preference for education is either too weak or too strong. Therefore, a society that values hard work and places a reasonable emphasis on education would achieve long-run growth.

The intuition of the above results can be explained as follows. First, a strong preference for leisure causes people to allocate too much time to leisure and too little time to production and education. As a result, the market size never becomes large enough for innovation to occur. Second, low education productivity gives rise to a low level of human capital, which reduces capacity for innovation. Third, low research productivity reduces incentives for innovation. Finally, a weak preference for education gives rise to a low level of human capital whereas a strong preference for education gives rise to overinvestment in human capital that crowds out resources for innovation.

This study relates to the literature on growth and innovation. The seminal study by Romer (1990) develops the R\&D-based growth model with the development of new products. ${ }^{1}$ While Romer (1990) and other early studies do not consider human capital accumulation in the R\&Dbased growth model, subsequent studies introduce human capital accumulation and explore its implications on innovation; see for example, Eicher (1996), Zeng (1997, 2003), Strulik (2005, 2007), Chu et al. (2013, 2016), Hashimoto and Tabata (2016) and Prettner and Strulik (2016). This study differs from them by considering human capital accumulation and innovation as the main engines of growth at different stages of development.

A number of studies also explore different stages of development in the R\&D-based growth model; see for example, Zilibotti (1995), Peretto (1999), Funke and Strulik (2000), Irmen (2005) and Kuwahara (2007, 2013, 2019). These studies consider the case in which an economy features human/physical capital accumulation in an early stage of development and then innovation in a later stage. Some of them also explore when this transition occurs and when the economy remains in the middle-income trap. ${ }^{2}$ This study complements these interesting studies by exploring how preferences for leisure and education affect the transition from capital accumulation to innovation.

Finally, this study also relates to the literature on preference formation and economic growth. Early studies by Galor and Moav (2002) and Doepke and Zilibotti (2008) develop interesting growth models with evolution in preferences, in which the proportion of individuals with different preferences for child quality in Galor and Moav (2002) and different preferences for patience and leisure in Doepke and Zilibotti (2008) evolves overtime and affects economic development.

\footnotetext{
${ }^{1}$ See also Aghion and Howitt (1992) who develop the Schumpeterian quality-ladder growth model.

${ }^{2}$ See Agenor (2017) for an excellent survey of studies on middle-income traps.
} 
A recent empirical study by Galor and Ozak (2016) provides evidence on the pre-industrial agricultural origins of differences in time preferences and their effects on education and technology in the modern era. Ashraf and Galor (2018) and Doepke and Zilibotti (2014) provide surveys of this literature. This study builds on this literature by exploring how differences in preferences affect an economy across stages of development with different growth engines and its likelihood of staying in a middle-income trap.

\section{The model}

We modify the Romer model to allow for a simple structure of overlapping generations and human capital accumulation. ${ }^{3}$ Individuals live for three periods. In her young age, an individual receives education. In her working age, the individual allocates her time between leisure, work and education of the next generation. In her old age, the individual consumes her saving.

\section{$2.1 \quad$ Individuals}

A unit continuum of individuals is born in each generation. The utility of an individual who works at time $t$ is given by

$$
U^{t}=u\left(l_{t}, C_{t+1}, H_{t+1}\right)=\eta \ln l_{t}+\ln C_{t+1}+\gamma \ln H_{t+1},
$$

where $l_{t}$ denotes the amount of time allocated to leisure at time $t$ and $\eta \geq 0$ is the leisure preference parameter. $C_{t+1}$ is consumption at time $t+1$. For simplicity, the individual only consumes in the old age. ${ }^{4} H_{t+1}$ denotes human capital the individual passes onto her child, and $\gamma>0$ is the education preference parameter. The individual allocates $e_{t}$ units of time to her child's education. The accumulation of human capital is determined as follows: ${ }^{5}$

$$
H_{t+1}=\phi e_{t}+(1-\delta) H_{t}
$$

where the parameter $\phi>0$ determines education productivity and the parameter $\delta \in(0,1)$ determines the depreciation of human capital that a generation passes onto the next. For simplicity, education is the only form of bequest. ${ }^{6}$

The individual allocates $1-l_{t}-e_{t}$ units of time to work and earns $w_{t}\left(1-l_{t}-e_{t}\right) H_{t}$ as real wage income. The individual devotes her entire wage income to saving at time $t$ and consumes the return at time $t+1$ :

$$
C_{t+1}=\left(1+r_{t+1}\right) w_{t}\left(1-l_{t}-e_{t}\right) H_{t},
$$

where $r_{t+1}$ is the real interest rate. Substituting (2) and (3) into (1), the individual maximizes

$$
\max _{e_{t}, l_{t}} U^{t}=\eta \ln l_{t}+\ln \left[\left(1+r_{t+1}\right) w_{t}\left(1-l_{t}-e_{t}\right) H_{t}\right]+\gamma \ln \left[\phi e_{t}+(1-\delta) H_{t}\right],
$$

\footnotetext{
${ }^{3}$ The formulation is based on Chu et al. (2016), who however focus on the second stage of development and do not consider the first stage.

${ }^{4}$ All our results hold if individuals also consume in the working age; see Appendix C.

${ }^{5}$ See Chu et al. (2016) for an extension that allows for public investment in education.

${ }^{6}$ See Chu et al. (2016) for an extension that allows for multiple channels of bequest.
} 
taking $\left\{r_{t+1}, w_{t}, H_{t}\right\}$ as given. The utility-maximizing levels of leisure $l_{t}$ and education $e_{t}$ are

$$
\begin{gathered}
l_{t}=\eta \frac{\phi+(1-\delta) H_{t}}{\phi(1+\eta+\gamma)}, \\
e_{t}=\frac{\phi \gamma-(1+\eta)(1-\delta) H_{t}}{\phi(1+\eta+\gamma)} .
\end{gathered}
$$

Substituting (5) into (2) yields the following law of motion for human capital:

$$
H_{t+1}=\frac{\gamma}{1+\eta+\gamma}\left[\phi+(1-\delta) H_{t}\right]
$$

Equation (6) shows that given any initial level $H_{0}$, human capital $H_{t}$ always converges to the following steady-state level:

$$
H^{*}=\frac{\phi \gamma}{1+\eta+\delta \gamma}
$$

\section{$2.2 \quad$ Final good}

This sector is characterized by perfect competition. Firms produce final good $Y_{t}$ (numeraire) using the following production function:

$$
Y_{t}=H_{Y, t}^{1-\alpha} \int_{0}^{N_{t}} X_{t}^{\alpha}(i) d i .
$$

$H_{Y, t}$ denotes human-capital-embodied production labor, and the parameter $\alpha \in(0,1)$ determines labor intensity $1-\alpha$ in production. There is a continuum of differentiated intermediate goods indexed by $i \in\left[0, N_{t}\right]$, and $X_{t}(i)$ denotes intermediate good $i$. Maximizing profit, we derive the conditional demand functions for $H_{Y, t}$ and $X_{t}(i)$ as

$$
\begin{gathered}
w_{t}=(1-\alpha) Y_{t} / H_{Y, t}, \\
p_{t}(i)=\alpha\left[H_{Y, t} / X_{t}(i)\right]^{1-\alpha} .
\end{gathered}
$$

\subsection{Intermediate goods}

This sector is characterized by monopolistic competition. A monopolistic firm produces $X_{t}(i)$ using a linear production function that transforms one unit of $Y_{t}$ into one unit of $X_{t}(i)$. The profit function is

$$
\pi_{t}(i)=p_{t}(i) X_{t}(i)-X_{t}(i)
$$

where the cost of producing one unit of $X_{t}(i)$ is one (recall that final good is the numeraire). The profit-maximizing price is

$$
p_{t}(i)=\frac{1}{\alpha}>1
$$

which is above the marginal cost of production. Substituting (12) into (10) shows that $X_{t}(i)=$ $X_{t}$ for all $i \in\left[1, N_{t}\right]$. Substituting (10) and (12) into (11) yields

$$
\pi_{t}=\left(\frac{1}{\alpha}-1\right) X_{t}=(1-\alpha) \alpha^{(1+\alpha) /(1-\alpha)} H_{Y, t} .
$$




\section{$2.4 \quad \mathrm{R} \& \mathrm{D}$}

This sector is characterized by perfect competition. Let $v_{t}$ denote the value of a newly invented intermediate good at the end of time $t$, which is given by the present value of future profits from time $t+1$ onwards:

$$
v_{t}=\sum_{s=t+1}^{\infty}\left[\pi_{s} / \prod_{\tau=t+1}^{s}\left(1+r_{\tau}\right)\right] .
$$

R\&D entrepreneurs devote $H_{R, t}$ units of human-capital-embodied labor to the invention of new products. The innovation process is specified as

$$
\Delta N_{t}=\theta N_{t} H_{R, t}
$$

where $\Delta N_{t} \equiv N_{t+1}-N_{t}$. The parameter $\theta>0$ determines R\&D productivity $\theta N_{t}$, where $N_{t}$ captures intertemporal knowledge spillovers as in Romer (1990). If the following holds:

$$
\Delta N_{t} v_{t}=w_{t} H_{R, t} \Leftrightarrow \theta N_{t} v_{t}=w_{t},
$$

then R\&D $H_{R, t}$ would be positive at time $t$. If $\theta N_{t} v_{t}<w_{t}$, then R\&D does not take place at time $t$ (i.e., $H_{R, t}=0$ ).

Lemma 1 RED $H_{R, t}$ is positive at time $t$ if and only if the following inequality holds:

$$
\left(1-l_{t}-e_{t}\right) H_{t}=\frac{\phi H_{t}+(1-\delta)\left(H_{t}\right)^{2}}{\phi(1+\eta+\gamma)}>\frac{1}{\theta}
$$

Proof. See Appendix A.

\subsection{Aggregation}

Imposing symmetry on (8) yields $Y_{t}=H_{Y, t}^{1-\alpha} N_{t} X_{t}^{\alpha}$. Then, substituting (10) and (12) into this equation yields

$$
Y_{t}=\alpha^{2 \alpha /(1-\alpha)} N_{t} H_{Y, t},
$$

which is the aggregate production function of $Y_{t}$. The resource constraint on final good is

$$
C_{t}=Y_{t}-N_{t} X_{t}=\left(1-\alpha^{2}\right) Y_{t}
$$

which uses $N_{t} X_{t}=\alpha^{2} Y_{t}$. Finally, the resource constraint on human-capital-embodied labor is

$$
\left(1-l_{t}-e_{t}\right) H_{t}=H_{Y, t}+H_{R, t} .
$$

\subsection{Equilibrium}

See Appendix B. 


\section{Stages of economic development}

In this section, we explore the two stages of economic development. In the first stage, the economy features only human capital accumulation. In the second stage, the economy features both human capital accumulation and innovation. The simplicity of the model allows us to derive a closed-form solution for the transition dynamics.

\subsection{Stage 1: Human capital accumulation only}

The initial level of human capital is $H_{0}$. We assume the following inequality holds at time 0 :

$$
\left(1-l_{0}-e_{0}\right) H_{0}=\frac{\phi H_{0}+(1-\delta)\left(H_{0}\right)^{2}}{\phi(1+\eta+\gamma)}<\frac{1}{\theta} .
$$

Then, Lemma 1 implies that $H_{R, 0}=0$ and

$$
H_{Y, 0}=\left(1-l_{0}-e_{0}\right) H_{0}=\frac{\phi H_{0}+(1-\delta)\left(H_{0}\right)^{2}}{\phi(1+\eta+\gamma)},
$$

where the second equality uses (4) and (5). At this stage of development, the economy features only human capital accumulation. Human capital $H_{t}$ accumulates according to the autonomous dynamics in (6). So long as the following inequality holds at time $t$ :

$$
\left(1-l_{t}-e_{t}\right) H_{t}=\frac{\phi H_{t}+(1-\delta)\left(H_{t}\right)^{2}}{\phi(1+\eta+\gamma)}<\frac{1}{\theta},
$$

we continue to have $H_{R, t}=0$ and

$$
H_{Y, t}=\left(1-l_{t}-e_{t}\right) H_{t}=\frac{\phi H_{t}+(1-\delta)\left(H_{t}\right)^{2}}{\phi(1+\eta+\gamma)} .
$$

Substituting (24) into (18) yields the level of output as

$$
Y_{t}=\alpha^{2 \alpha /(1-\alpha)} N_{0} \frac{\phi H_{t}+(1-\delta)\left(H_{t}\right)^{2}}{\phi(1+\eta+\gamma)},
$$

where $N_{0}$ remains at the initial level and output $Y_{t}$ increases as human capital $H_{t}$ accumulates.

Human capital $H_{t}$ eventually converges to $H^{*}$ in (7). Substituting (7) into (23) yields

$$
\left(1-l^{*}-e^{*}\right) H^{*}=\frac{\phi \gamma}{(1+\eta+\delta \gamma)^{2}}<\frac{1}{\theta}
$$

If the inequality in (26) holds, then the economy would never experience innovation. The economy remains indefinitely in this middle-income trap in the case of strong leisure preference $\eta$, low research productivity $\theta$, low education productivity $\phi$, or education preference $\gamma$ being too weak or too strong. Intuitively, strong leisure preference causes people to allocate too much time to leisure and too little time to education, whereas low education productivity and low research productivity reduce incentives for human capital accumulation and innovation. A 
weak preference for education causes a low level of human capital whereas a strong preference for education leads to overinvestment in human capital that crowds out the amount of humancapital-embodied labor $\left(1-l^{*}-e^{*}\right) H^{*}$ for innovation.

In the middle-income trap, the steady-state level of output is given by

$$
Y^{*}=\alpha^{2 \alpha /(1-\alpha)} N_{0} \frac{\phi \gamma}{(1+\eta+\delta \gamma)^{2}},
$$

which is decreasing in leisure preference $\eta$, increasing in education productivity $\phi$, and an inverted- $U$ function in education preference $\gamma$. Intuitively, strong leisure preference causes people to allocate too much time to leisure and too little time to production, whereas low education productivity reduces incentives for human capital accumulation. Finally, as before, a weak preference for education leads to a low level of human capital whereas a strong preference for education leads to overinvestment in human capital that crowds out the amount of humancapital-embodied labor $\left(1-l^{*}-e^{*}\right) H^{*}$ for production.

Proposition 1 If (1) leisure preference $\eta$ is sufficiently strong, (2) research productivity $\theta$ or education productivity $\phi$ is sufficiently low, or (3) education preference $\gamma$ is sufficiently weak or sufficiently strong, the economy would remain in the middle-income trap, in which steady-state output $Y^{*}$ is decreasing in leisure preference $\eta$, increasing in education productivity $\phi$, and an inverted- $U$ function in education preference $\gamma$.

Proof. Use (26) and (27).

\subsection{Stage 2: Innovation and human capital accumulation}

Lemma 1 implies that if the following inequality holds:

$$
\left(1-l^{*}-e^{*}\right) H^{*}=\frac{\phi \gamma}{(1+\eta+\delta \gamma)^{2}}>\frac{1}{\theta}
$$

then human capital $H_{t}$ eventually becomes sufficiently large to trigger the activation of innovation. This threshold $\widetilde{H}$ is given by

$$
\frac{\phi \widetilde{H}+(1-\delta)(\widetilde{H})^{2}}{\phi(1+\eta+\gamma)}=\frac{1}{\theta} \Leftrightarrow \widetilde{H}=\frac{-\phi+\sqrt{\phi^{2}+4(1-\delta)(1+\eta+\gamma) \phi / \theta}}{2(1-\delta)} \in\left(H_{0}, H^{*}\right) .
$$

Therefore, for $H_{t}>\widetilde{H}$, the R\&D condition in (16) holds and R\&D $H_{R, t}$ is positive.

Substituting (18) into (9) yields the equilibrium wage rate as

$$
w_{t}=(1-\alpha) \alpha^{2 \alpha /(1-\alpha)} N_{t} .
$$

Then, substituting (30) into (16) yields the equilibrium invention value as

$$
v_{t}=\frac{(1-\alpha) \alpha^{2 \alpha /(1-\alpha)}}{\theta} .
$$


The structure of overlapping generations implies that the value of assets at the end of time $t$ must equal the amount of saving at time $t$ given by wage income at time $t$ :

$$
N_{t+1} v_{t}=w_{t}\left(1-l_{t}-e_{t}\right) H_{t}=w_{t}\left(H_{Y, t}+H_{R, t}\right)
$$

where the second equality uses (20). Substituting (30) and (31) into (32) yields

$$
N_{t+1}=\theta N_{t}\left(H_{Y, t}+H_{R, t}\right) .
$$

Combining (15) and (33) yields the equilibrium level of $H_{Y, t}$ as

$$
H_{Y, t}=\frac{1}{\theta}
$$

for all $t$. Substituting (4), (5) and (34) into (20) yields the equilibrium level of $H_{R, t}$ as

$$
H_{R, t}=\left(1-l_{t}-e_{t}\right) H_{t}-H_{Y, t}=\frac{\phi H_{t}+(1-\delta)\left(H_{t}\right)^{2}}{\phi(1+\eta+\gamma)}-\frac{1}{\theta} .
$$

Substituting (35) into (15) yields the equilibrium growth rate of $N_{t}$ as

$$
g_{t} \equiv \frac{\Delta N_{t}}{N_{t}}=\theta H_{R, t}=\frac{\theta}{\phi(1+\eta+\gamma)}\left[\phi H_{t}+(1-\delta)\left(H_{t}\right)^{2}\right]-1 .
$$

For a given $H_{t}, g_{t}$ is increasing in research productivity $\theta$ but decreasing in leisure preference $\eta$, education productivity $\phi$ and education preference $\gamma$. Intuitively, high research productivity raises incentives for innovation whereas high education productivity or strong education preference leads to a reallocation of resources from innovation to education. Finally, strong leisure preference causes people to allocate too much time to leisure and too little time to innovation.

The growth rate $g_{t}$ also determines output growth. To see this, we substitute (34) into (18) to derive the equilibrium level of output as

$$
Y_{t}=\frac{\alpha^{2 \alpha /(1-\alpha)}}{\theta} N_{t},
$$

which grows at the same rate as $N_{t}$. Therefore, although human capital continues to accumulate until reaching the steady state, human capital accumulation affects the growth rate of output only indirectly via innovation. As human capital $H_{t}$ increases according to (6), the equilibrium growth rate $g_{t}$ in (36) also increases.

As human capital converges to its steady-state level $H^{*}$ in $(7)$, the equilibrium growth rate also converges to its steady state given by

$$
g^{*}=\frac{\theta \phi \gamma}{(1+\eta+\delta \gamma)^{2}}-1
$$

which is decreasing in leisure preference $\eta$, increasing in research productivity $\theta$ and education productivity $\phi$, and an inverted- $U$ function in education preference $\gamma \cdot{ }^{7}$ Intuitively, strong leisure preference causes people to allocate too much time to leisure and too little time to innovation and education, whereas low education productivity reduces incentives for human capital accumulation and low research productivity reduces incentives for innovation. Finally, a weak preference for education leads to a low level of human capital whereas a strong preference for education leads to overinvestment in human capital that crowds out resources for innovation.

\footnotetext{
${ }^{7}$ This inverted-U effect of education preference on steady-state growth was also shown in Chu et al. (2016).
} 
Proposition 2 In the second stage of development, innovation is activated. For a given level of human capital $H_{t}$, the equilibrium growth rate $g_{t}$ is increasing in research productivity $\theta$ but decreasing in leisure preference $\eta$, education productivity $\phi$ and education preference $\gamma$. As human capital $H_{t}$ accumulates, the equilibrium growth rate $g_{t}$ increases. As human capital $H_{t}$ converges to the steady state, the equilibrium growth rate $g_{t}$ also converges to the steady-state growth rate $g^{*}$, which is decreasing in leisure preference $\eta$, increasing in research productivity $\theta$ and education productivity $\phi$, and an inverted- $U$ function in education preference $\gamma$.

Proof. Use (36) and (38).

\section{Conclusion}

In this study, we have developed a simple growth model that features two stages of development to explore the effects of preferences for leisure and education on the transition from human capital accumulation to innovation. We find that under a strong preference for leisure or a parental preference for education that is either too weak or too strong, the economy would remain in a middle-income trap with a steady-state level of output. Only when a society values hard work and places a reasonable emphasis on education, the economy would achieve economic growth in the long run.

\section{References}

[1] Agenor, P.-R., 2017. Caught in the middle? The economics of middle-income traps. Journal of Economic Surveys, 31, 771-791.

[2] Aghion, P., and Howitt, P., 1992. A model of growth through creative destruction. Econometrica, 60, 323-351.

[3] Ashraf, Q., and Galor, O., 2018. The macrogenoeconomics of comparative development. Journal of Economic Literature, 56, 1119-1155.

[4] Chu, A., Cozzi, G., and Liao, C., 2013. Endogenous fertility and human capital in a Schumpeterian growth model. Journal of Population Economics, 26, 181-202.

[5] Chu, A., Furukawa, Y., and Zhu, D., 2016. Growth and parental preference for education in China. Journal of Macroeconomics, 49, 192-202.

[6] Doepke, M., and Zilibotti, F., 2008. Occupational choice and the spirit of capitalism. Quarterly Journal of Economics, 123, 747-793.

[7] Doepke, M., and Zilibotti, F., 2014. Culture, entrepreneurship, and growth. Handbook of Economic Growth, 2, 1-48.

[8] Eicher, T., 1996. Interaction between endogenous human capital and technological change. Review of Economic Studies, 63, 127-44. 
[9] Funke, M., and Strulik, H., 2000. On endogenous growth with physical capital, human capital and product variety. European Economic Review, 44, 491-515.

[10] Galor, O., and Moav, O., 2002. Natural selection and the origin of economic growth. Quarterly Journal of Economics, 117, 1133-1191.

[11] Galor, O., and Ozak, O., 2016. The agricultural origins of time preference. American Economic Review, 106, 3064-3103

[12] Hashimoto, K., and Tabata, K., 2016. Demographic change, human capital accumulation and R\&D-based growth. Canadian Journal of Economics, 49, 707-737.

[13] Irmen, A., 2005. Extensive and intensive growth in a neoclassical framework. Journal of Economic Dynamics \& Control, 29, 1427-1448.

[14] Kuwahara, S., 2007. The mechanics of economic growth through capital accumulation and technological progress. Japanese Economic Review, 58, 504-523.

[15] Kuwahara, S., 2013. Dynamical analysis of the R\&D-based growth model with a regime switch. Journal of Economics, 108, 35-57.

[16] Kuwahara, S., 2019. Multiplicity and stagnation under the Romer model with increasing returns of R\&D. Economic Modelling, 79, 86-97.

[17] Peretto, P., 1999. Industrial development, technological change, and long-run growth. Journal of Development Economics, 59, 389-417.

[18] Prettner, K., and Strulik, H., 2016. Technology, trade, and growth: The role of education. Macroeconomic Dynamics, 20, 1381-1394.

[19] Romer, P., 1990. Endogenous technological progress. Journal of Political Economy, 98, S71-S102.

[20] Strulik, H., 2005. The role of human capital and population growth in R\&D-based models of economic growth. Review of International Economics, 13, 129-145.

[21] Strulik, H., 2007. Too much of a good thing? The quantitative economics of R\&D-driven growth revisited. Scandinavian Journal of Economics, 109, 369-386.

[22] Zeng, J., 1997. Physical and human capital accumulation, R\&D and economic growth. Southern Economic Journal, 63, 1023-1038.

[23] Zeng, J., 2003. Reexamining the interactions between innovation and capital accumulation. Journal of Macroeconomics, 25, 541-560.

[24] Zilibotti, F., 1995. A Rostovian model of endogenous growth and underdevelopment traps. European Economic Review, 39, 1569-1602. 


\section{Appendix A: Proof of Lemma 1}

Proof of Lemma 1. If (17) holds, then (35) shows that $H_{R, t}>0$. Now, let's consider the case in which

$$
\left(1-l_{t}-e_{t}\right) H_{t}=\frac{\phi H_{t}+(1-\delta)\left(H_{t}\right)^{2}}{\phi(1+\eta+\gamma)}<\frac{1}{\theta} .
$$

Recall that the value of assets at the end of time $t$ must equal the amount of saving at time $t$ given by wage income at time $t$ such that

$$
N_{t+1} v_{t}=w_{t}\left(1-l_{t}-e_{t}\right) H_{t}
$$

Substituting (A2) into (A1) yields

$$
w_{t}>\theta N_{t+1} v_{t} \geq \theta N_{t} v_{t}
$$

where the second inequality uses $N_{t+1} \geq N_{t}$. Equation (A3) implies that $\Delta N_{t} v_{t}=w_{t} H_{R, t}$ in (16) cannot hold unless $H_{R, t}=0$. 


\section{Appendix B: Equilibrium}

The equilibrium is a sequence of allocations $\left\{X_{t}(i), Y_{t}, C_{t}, H_{Y, t}, H_{R, t}, H_{t}, e_{t}, l_{t}\right\}$ and prices $\left\{p_{t}(i), w_{t}, r_{t}, v_{t}\right\}$ that satisfy the following conditions:

- individuals choose $\left\{e_{t}, l_{t}\right\}$ to maximize utility taking $\left\{r_{t+1}, w_{t}, H_{t}\right\}$ as given;

- competitive firms produce $Y_{t}$ to maximize profit taking $\left\{p_{t}(i), w_{t}\right\}$ as given;

- a monopolistic firm produces $X_{t}(i)$ and chooses $p_{t}(i)$ to maximize profit;

- competitive entrepreneurs perform R\&D to maximize profit taking $\left\{w_{t}, v_{t}\right\}$ as given;

- the market for final good clears such that $Y_{t}=N_{t} X_{t}+C_{t}$;

- the market for human-capital-embodied labor clears such that $H_{Y, t}+H_{R, t}=\left(1-l_{t}-e_{t}\right) H_{t}$;

- the amount of saving equals the value of assets such that $w_{t}\left(1-l_{t}-e_{t}\right) H_{t}=N_{t+1} v_{t}$. 


\section{Appendix C: Consumption}

Suppose we modify (1) to allow for consumption also in the working age. Then, we have

$$
U^{t}=u\left(l_{t}, C_{t}^{t}, C_{t+1}^{t}, H_{t+1}\right)=\eta \ln l_{t}+(1-\beta) \ln C_{t}^{t}+\beta \ln C_{t+1}^{t}+\gamma \ln H_{t+1},
$$

where $\beta \in(0,1)$ determines the relative importance of $C_{t}^{t}$ and $C_{t+1}^{t}$, which denote consumption at time $t$ and $t+1$ of an individual who works at time $t$. Let $s_{t}$ denote the saving rate. Then,

$$
\begin{gathered}
C_{t}^{t}=\left(1-s_{t}\right) w_{t}\left(1-l_{t}-e_{t}\right) H_{t}, \\
C_{t+1}^{t}=\left(1+r_{t+1}\right) s_{t} w_{t}\left(1-l_{t}-e_{t}\right) H_{t} .
\end{gathered}
$$

Substituting (2), (C2) and (C3) into (C1) and maximizing utility yield $s_{t}=\beta$ and also (4)-(6) as before. The rest of the model is the same as before.

The value of assets at the end of time $t$ must equal the amount of saving at time $t$ :

$$
N_{t+1} v_{t}=s_{t} w_{t}\left(1-l_{t}-e_{t}\right) H_{t}=s_{t} w_{t}\left(H_{Y, t}+H_{R, t}\right),
$$

which differs from (32) due to $s_{t}=\beta<1$. Substituting (30) and (31) into (C4) yields

$$
N_{t+1}=\beta \theta N_{t}\left(H_{Y, t}+H_{R, t}\right) .
$$

Substituting (4) and (5) into (20) yields

$$
H_{Y, t}+H_{R, t}=\left(1-l_{t}-e_{t}\right) H_{t}=\frac{\phi H_{t}+(1-\delta)\left(H_{t}\right)^{2}}{\phi(1+\eta+\gamma)} .
$$

Combining (15), (C5) and (C6) yields

$$
H_{R, t}=\beta \frac{\phi H_{t}+(1-\delta)\left(H_{t}\right)^{2}}{\phi(1+\eta+\gamma)}-\frac{1}{\theta},
$$

where I have assumed $H_{R, t}>0$, which holds if and only if

$$
\beta \frac{\phi H_{t}+(1-\delta)\left(H_{t}\right)^{2}}{\phi(1+\eta+\gamma)}>\frac{1}{\theta} .
$$

In the first stage of development, we have $H_{R, t}=0$ and

$$
H_{Y, t}=\left(1-l_{t}-e_{t}\right) H_{t}=\frac{\phi H_{t}+(1-\delta)\left(H_{t}\right)^{2}}{\phi(1+\eta+\gamma)} .
$$

The economy is in this stage whenever $H_{t}<\widetilde{H}$, where $\widetilde{H}$ is given by

$$
\beta \frac{\phi \widetilde{H}+(1-\delta)(\widetilde{H})^{2}}{\phi(1+\eta+\gamma)}=\frac{1}{\theta} \Leftrightarrow \widetilde{H}=\frac{-\phi+\sqrt{\phi^{2}+4(1-\delta)(1+\eta+\gamma) \phi /(\beta \theta)}}{2(1-\delta)} .
$$


The economy remains in this middle-income trap indefinitely if

$$
\beta\left(1-l^{*}-e^{*}\right) H^{*}=\frac{\beta \phi \gamma}{(1+\eta+\delta \gamma)^{2}}<\frac{1}{\theta},
$$

which holds if $\beta$ is sufficiently small (i.e., individuals are impatient).

In the second stage of development, we have $H_{R, t}>0$ and

$$
g_{t} \equiv \frac{\Delta N_{t}}{N_{t}}=\theta H_{R, t}=\frac{\beta \theta}{\phi(1+\eta+\gamma)}\left[\phi H_{t}+(1-\delta)\left(H_{t}\right)^{2}\right]-1
$$

which is increasing in $\beta$ and determined by the dynamics of $H_{t}$ in (6) as before. As $H_{t}$ converges to its steady state $H^{*}$ in $(7)$, the growth rate $g_{t}$ also converges to its steady state given by

$$
g^{*}=\frac{\beta \theta \phi \gamma}{(1+\eta+\delta \gamma)^{2}}-1
$$

which shows that $g^{*}$ is increasing in $\beta$ and other comparative statics are the same as before. 